\title{
SMALL ANGLE X-RAY SCATTERING STUDIES OF AGGREGATION IN SUPERCRITICAL FLUID SOLUTIONS
}

\author{
J. L. Fulton \\ D. M. Pfund
}

October 1994

Presented at the

Third International Symposium on Supercritical

Fluids Conference

October 17-19, 1994

Strasbourg, France

Prepared for

the U.S. Department of Energy

under Contract DE-AC06-76RLO 1830

Pacific Northwest Laboratory

Richland, Washington 99352

This report was prepared as an account of work sponsored by an agency of the United States Government. Neither the United States Government nor any agency thereof, nor any of their employees, makes any warranty, express or implied, or assumes any legal liability or responsibility for the accuracy, completeness, or usefulness of any information, apparatus, product, or process disclosed, or represents that its use would not infringe privately owned rights. Reference herein to any specific commercial product, process, or service by trade name, trademark, manufacturer, or otherwise does not necessarily constitute or imply its endorsement, recommendation, or favoring by the United States Government or any agency thereof. The views and opinions of authors expressed herein do not necessarily state or reflect those of the United States Government or any agency thereof. 


\section{DISCLAIMER}

Portions of this document may be illegible in electronic image products. Images are produced from the best available original document. 


\section{SUMMARY}

- Small-angle x-ray scattering (SAXS) can be used to derive structural information on molecular aggregates having sizes from 2 to $200 \mathrm{~nm}$. Not only is the technique useful for probing fluid structure in pure and simple binary supercritical fluid systems, but the technique is also well suited to investigate a range of much more complex multi-molecular aggregates that form when surfactants are added to supercritical fluids. We will describe the experimental apparatus that was constructed for these studies and the experimental approach used to collect the scattering data. We present scattering results for pure fluids and for fluids containing various types of microemulsion phases, including reverse micelle and normal micelle phases. These results demonstrate that SAXS is a powerful technique for probing various types of molecular aggregation in supercritical fluid solutions.

\section{INTRODUCTION}

Supercritical fluid solutions contain an intriguing array of molecular aggregation processes. These aggregates can consist of simple solute/solvent clusters that form near the critical point of the solution, or they can consist of much larger structures formed upon addition of surfactants or polymers to a supercritical fluid. Small-angle scattering techniques $(1,2)$ offer the potential to fully describe the spatial distribution of these aggregating molecules in a supercritical solution. Such information is essential in developing models to describe solvation in supercritical fluids as well as in providing insights into the mechanisms of aggregation. Recent application of small-angle scattering methods to near-critical and supercritical fluids attest to the utility of the technique $(3,4,5,6)$.

Both small-angle $\mathrm{x}$-ray and neutron scattering (SANS) provide analogous information on fluid microstructure. Table 1 provides a comparison of the advantages of these techniques relative to their application to supercritical fluid systems. The theoretical methods required to interpret the scattering information are virtually identical, except for the basis of the scattering contrast: for SAXS, inhomogeneities in the electron densities give rise to coherent, excess scattering, whereas inhomogeneities in the nucleus's scattering cross section give rise to excess neutron scattering. Both methods require a high-pressure cell with beam-transparent windows. For examination of small molecular clusters, neutron scattering perhaps holds an advantage since the scattering contrast can be finely controlled through variation of the percent deuteration of the solute or solvent. (Protons and deuterons have very different scattering cross sections.) For studies of organic liquid solutions, SAXS is generally not the preferred method because of the poor electron density contrast between the solute and solvent. For supercritical fluids, however, this problem is largely eliminated since the lower-density supercritical fluid phase has 
good electron-density contrast with the higher-density solutes. A further advantage of SAXS is the relatively short time required to obtain a single spectrum. As a result, many experiments can be run sequentially in a single high pressure cell. Clearly, both SAXS and SANS are powerful techniques to probe structure in supercritical fluid solutions. With recent refinements in theory and experimental methods and the increased availability of scattering facilities, small angle scattering is becoming a routine technique.

To demonstrate the utility of SAXS for supercritical fluids solutions, we present results for a variety of different microstructured systems

\section{Table 1. A Comparison of SAXS and SANS Methods for Fluids}

\begin{tabular}{l|cc}
\multicolumn{1}{c}{ Property } & X-rays & Neutrons \\
\hline Scattering contrast & Electron density & Nucleus Prop. \\
Theoretical interpretation of scattering curves & No difference & No difference \\
Availability of facilities & Good & Fair \\
Cell design and experimental & Special cell & Special cell \\
Very small molecular clusters & Fair & Good \\
Larger molecular aggregates & Good & Good \\
Supercritical fluids contrast & Good & Good \\
Integration times & $5-10 \mathrm{~min}$ & $\sim 1 \mathrm{~h}$
\end{tabular}

\section{EXPERIMENTAL}

A schematic of the scattering cell and data collection technique is shown in Figure 1. The small-angle scattering cell is composed of a block of 316 stainless steel containing windows for transmission of the $\mathrm{X}$-ray beam and an optical view window. The maximum operating pressure of this design was 500 bar. The sample volume of the scattering cell is about $10 \mathrm{ml}$, and the contents of the cell can be stirred with a $10-$ x $3-\mathrm{mm}$, Teflon-coated, magnetically-coupled stir bar while the cell is mounted on the beamline goniometer.

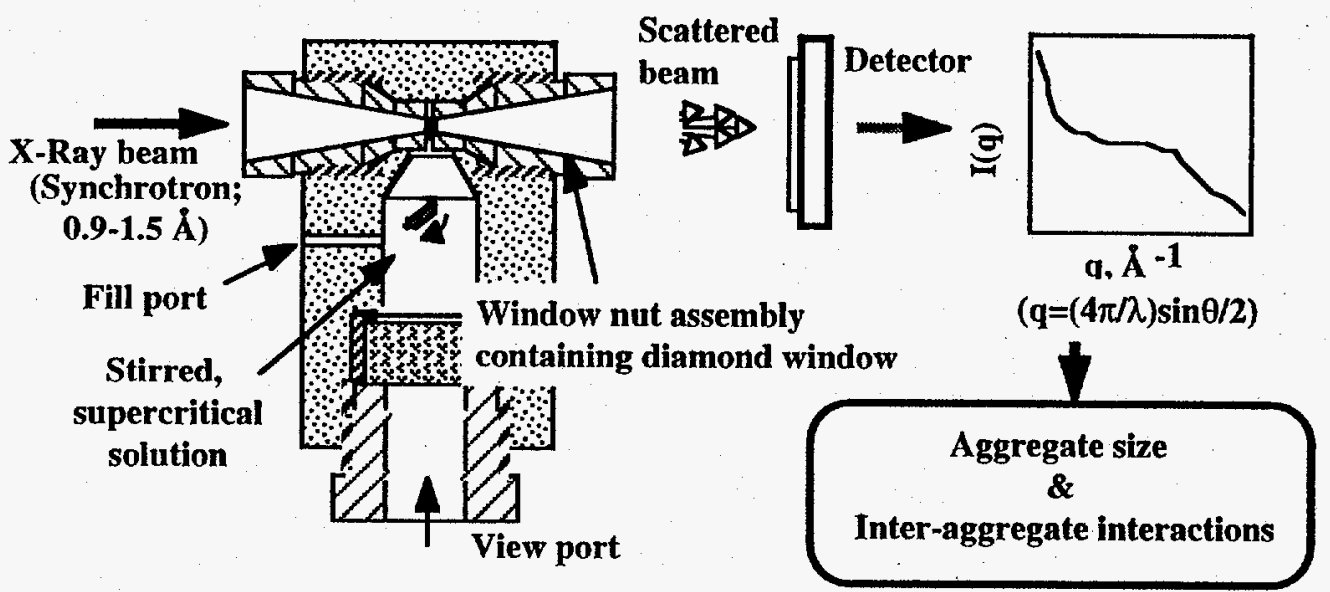

Figure 1. The high-pressure, small-angle $x$-ray scattering cell and the associated datacollection and analysis techniques.

The view-port window contained a sapphire window that was sealed to the metal block by a 2.54-cm-diameter gold-plated metal V-ring seal (Parker, No. 8812-2001-0050). This window provides a means of viewing the sample to determine the number of phases. The $x$-ray transmission windows consist of small diamond windows ( $3 \mathrm{~mm}$ diameter $\times 0.5 \mathrm{~mm}$ thick) 
that are brazed to the tip of a standard 1/4-in. high-pressure fitting (Assembly supplied by Omley Industries, Bend Oregon, USA). X-ray pathlengths for the xenon solutions were 0.1 $\mathrm{mm}$ and were $2.4 \mathrm{~mm}$ for all other samples. The percent transmission of the beam through these windows depends upon the $\mathrm{x}$-ray wavelength, $\lambda$. For $\lambda<1.0 \AA$, transmission is greater than $90 \%$. For the commonly used copper-edge wavelength of $1.38 \AA$, about $50 \%$ transmission is seen. Since synchrotron photon fluxes at this wavelength are very high, very low noise scattering spectra, spanning 4 orders-of-magnitude in scattering intensity, can be obtained using 10-minute integration times.

The $\mathrm{x}$-ray diffraction (SAXS) data were acquired on the Time-Resolved Diffraction Facility (station X12B) at the National Synchrotron Light Source (Brookhaven National Laboratory), using a custom-built 2-dimensional gas delay-line detector $(10 \mathrm{~cm} \mathrm{x} 10 \mathrm{~cm}, 512 \mathrm{x}$ 512 pixels), interfaced to a real-time histogramming memory system. The optical system provides a doubly-focused (spot size: $0.5-$ x 0.5 -mm FWHM) monochromatic $x$-ray beam (bandpass: $\sim 5 \times 10^{-4} \Delta \lambda / \lambda$ ) spanning 0.9 to $1.5 \AA$. The maximum forward scattering angle, $5^{\circ}$ from incident, was limited by the cone angle on the diamond window-nut assembly that gives a maximum available $q$ range $\{$ where $q=(4 \pi / \lambda) \sin (\theta / 2)\}$ approaching the wide angle scattering region of $0.6 \AA^{-1}$ for $\lambda=0.9 \AA$. Before comparison, the results from separate experiments made under different sample and instrument conditions were scaled to remove differences due to detector non-uniformity, beam intensity, and sample transmission.

Sample preparation usually involves introducing the liquid or solid solute directly into the scattering cell. Scattering measurements were performed by starting with the highest-density solution and then discharging small amounts of the solution to obtain progressively lower pressures. Through this technique, the overall mole fraction of the solute remained constant. Fluid pressure was monitored to \pm 1 bar with an electronic transducer (Precise Sensors, Inc., No. C451), which was calibrated against a deadweight tester (Ashcroft, No. 1305-D). The temperature of the SAXS cell was controlled using a three-mode controller with a platinum resistance probe (Omega, No. N2001) to an accuracy of $\pm 0.2^{\circ} \mathrm{C}$.

\section{INTERPRETATION OF SCATTERING CURVES}

Many approaches exist for treating scattering data, ranging from simple approximations to exact treatments involving direct coupling to theory or simulation. In the section that follows, we briefly describe three of these methods.

An approximation used to determine the average size of the density fluctuations near the critical point of the solution is the Ornstein-Zernicke-Debye relation for scattering at small angles:

$$
\frac{1}{I(q)} \approx \frac{1}{I(0)}\left(1+\frac{L^{2}}{6} q^{2}\right)
$$

where $I(q)$ is the total excess x-ray scattering, $L$ is the correlation length of the density fluctuations, and $q$ is the magnitude of the scattering vector given by $q=(4 \pi / \lambda) \sin (\theta / 2)$, with $\lambda$ and $\theta$ the $\mathrm{x}$-ray wavelength and scattering angle, respectively.

Another useful approximation for the low $q$ region is the Guinier approximation yielding radii of gyration, $R_{g}$, for most types of molecular aggregates:

$$
\ln [I(q)]-\ln [I(0)]=q^{2} R_{g}^{2} / 3
$$

For systems containing larger aggregates, a more exact treatment of the scattering data involves contributions to the total excess $\mathrm{x}$-ray scattering, $I(q)$, arising from two sources: 1 ) the particle contributions, $P(q)$, which depend solely upon the size and shape of the particle and 2) an appropriate structure factor, $S(q)$, which accounts for attractive or repulsive 
interactions between particles. By assuming that the solvent is structureless, the total scattering is simply

$$
I(q)=\rho S(q) P(q)
$$

where $\rho$ is the particle number density. When the system is sufficiently dilute, interparticle interactions are significantly reduced, yielding $S(q)=1$.

An example of $P(q)$ for a monodisperse system of homogeneous spheres of radius $r$, is given by

$$
P(q)=P(0)\left[3 \frac{\sin (q r)-q r \cos (q r)}{(q r)^{3}}\right]^{2}
$$

The more general treatments of scattering theory based upon integral equations or molecular dynamics are beyond the scope of this paper.

\section{RESULTS AND DISCUSSION}

Figures 2-5 show the application of SAXS to a variety of supercritical fluid solutions that contain aggregating species. In Figure 2, the correlation lengths are determined for two different systems near their critical points. For pure xenon, near the critical density but about $10^{\circ} \mathrm{C}$ above the critical temperature, there are appreciable density fluctuations corresponding to a correlation length, $L$, of $30.3 \AA$. The xenon/helium system is much closer to $T_{\mathcal{C}}$, leading to much larger density fluctuations corresponding to $L=57.8 \AA$.

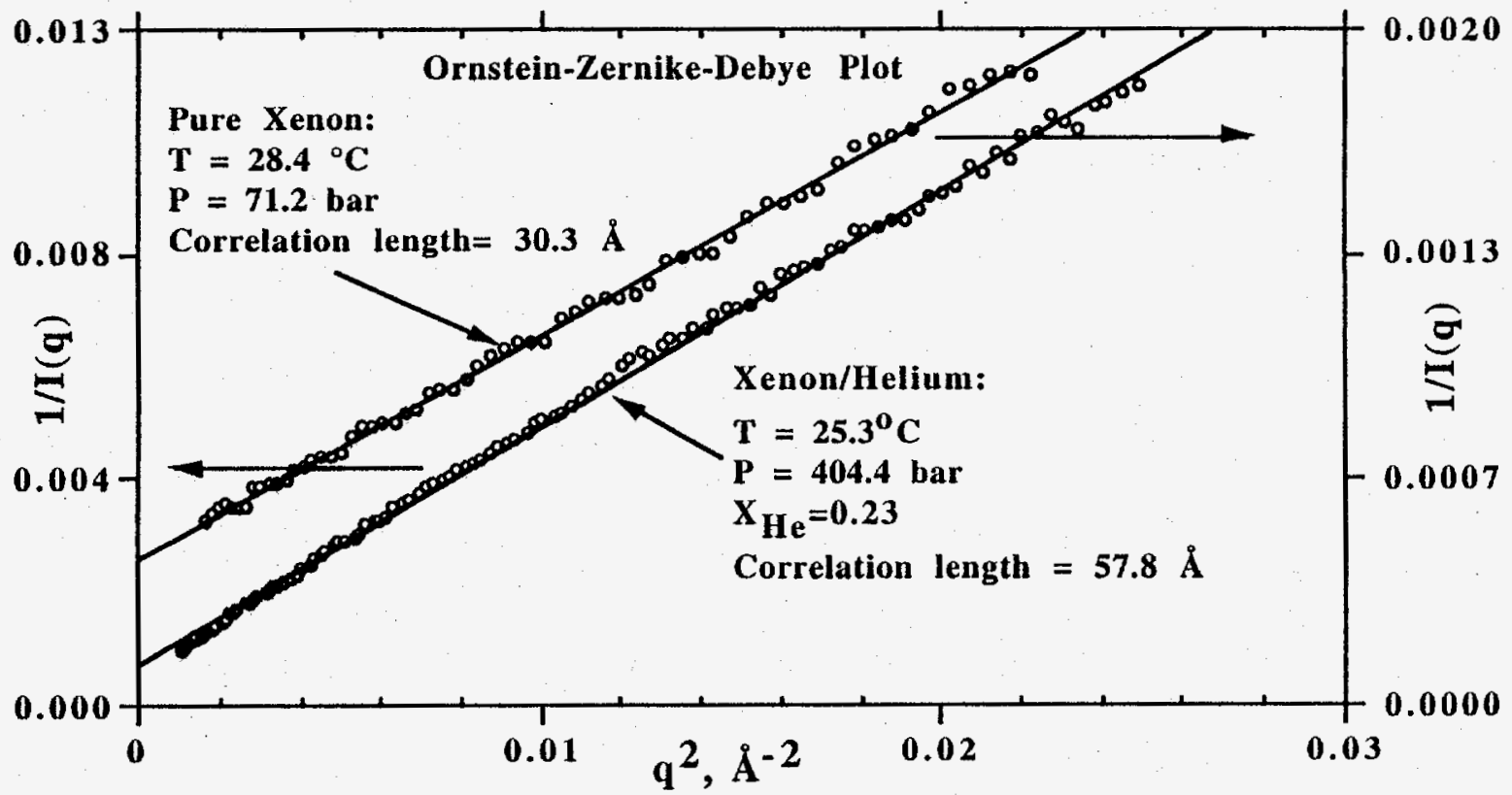

Figure 2. Ornstein-Zernicke-Debye plot for supercritical xenon and a xenon/helium mixture with correlation lengths of 30.3 and $57.8 \AA$, respectively.

In another treatment of the pure xenon data, shown in Figure 3, the scattering data are modeled using an integral equation approach known as the Hybrid Mean Spherical Approximation. The characteristic behavior of increased density fluctuations near the critical density and the disappearance of these fluctuations at high pressures are well treated by this model. 


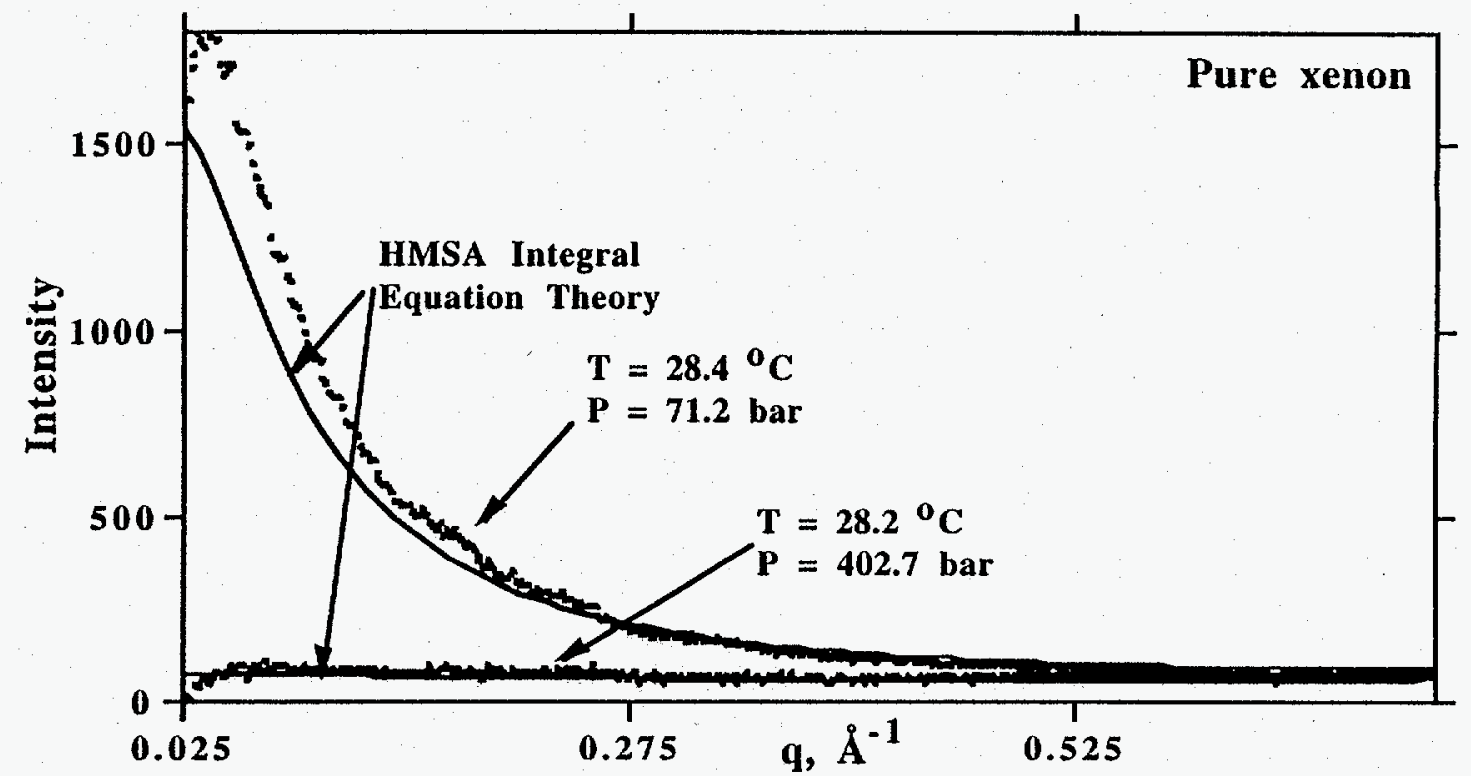

Figure 3. SAXS spectrum of pure xenon at moderate and high density. The lines represent a fit based upon an integral equation treatment of the Hybrid Mean Spherical Approximation.

Certain surfactants form molecular aggregates in supercritical fluids containing over 100 molecules per aggregate. These microemulsions, as they are known, are clear, thermodynamically stable solutions generally containing water, a surfactant, and an oil (i.e., a nonpolar or low polarity fluid). The surfactant molecules form organized molecular aggregates and preferentially occupy the interface between the oil and water phases. Microemulsions allow highly polar organic and inorganic salts to be dissolved in nonpolar supercritical fluid phases. Figure 4 shows a SAXS spectrum for a mixture of a surfactant (AOT, bis(2ethylhexyl)sulfosuccinate sodium salt) and supercritical xenon. For this dilute system at high pressure, the interparticle structure factor, $S(q) \approx 1$. In this case, $P(q)$, defined by Equation 3 , provides a good fit to the scattering data for spheres having a diameter of $3.4 \mathrm{~nm}$.

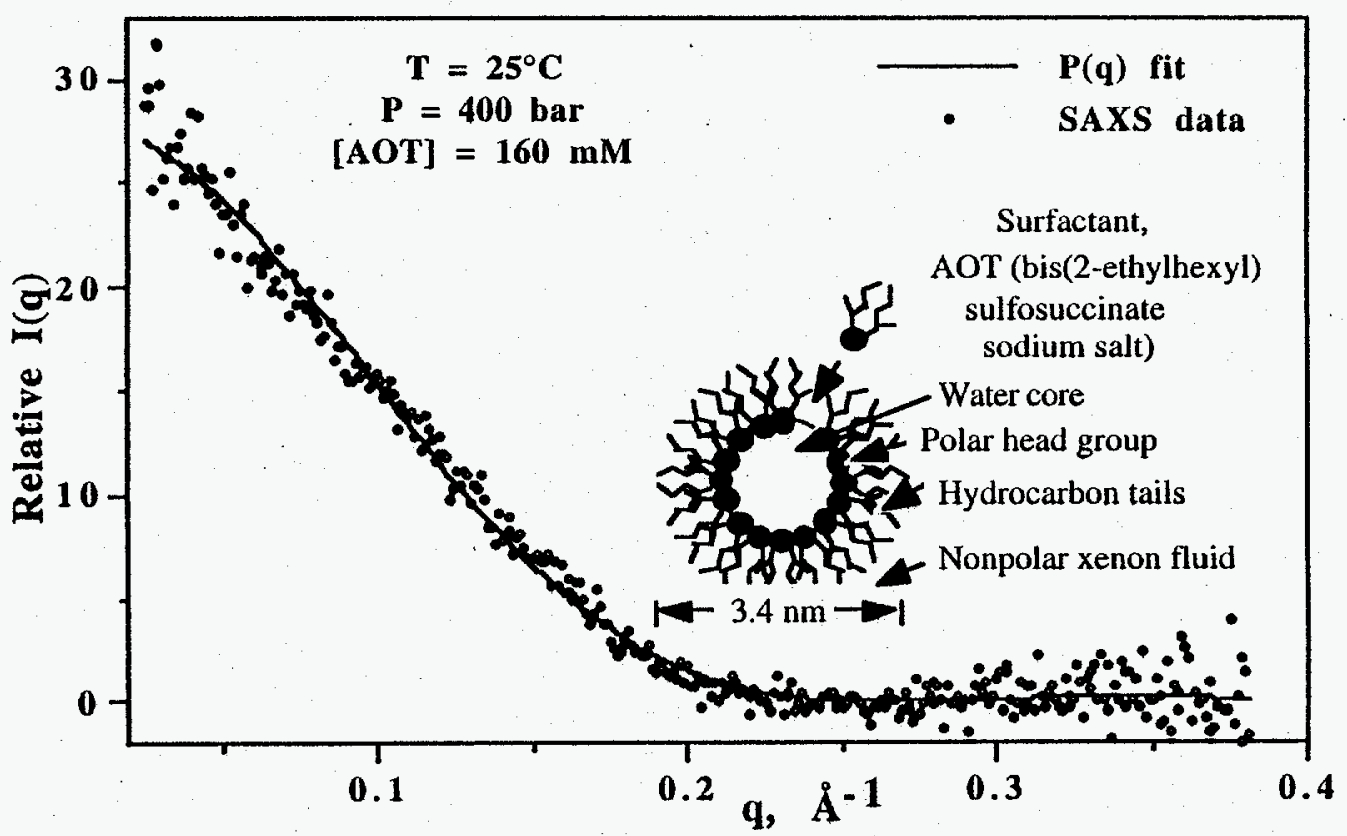

Figure 4. SAXS spectrum of reverse micelle phase formed in supercritical xenon and the fit to a model of a spherical-shaped aggregate. The diameter of the surfactant aggregate is $3.4 \mathrm{~nm}$. 


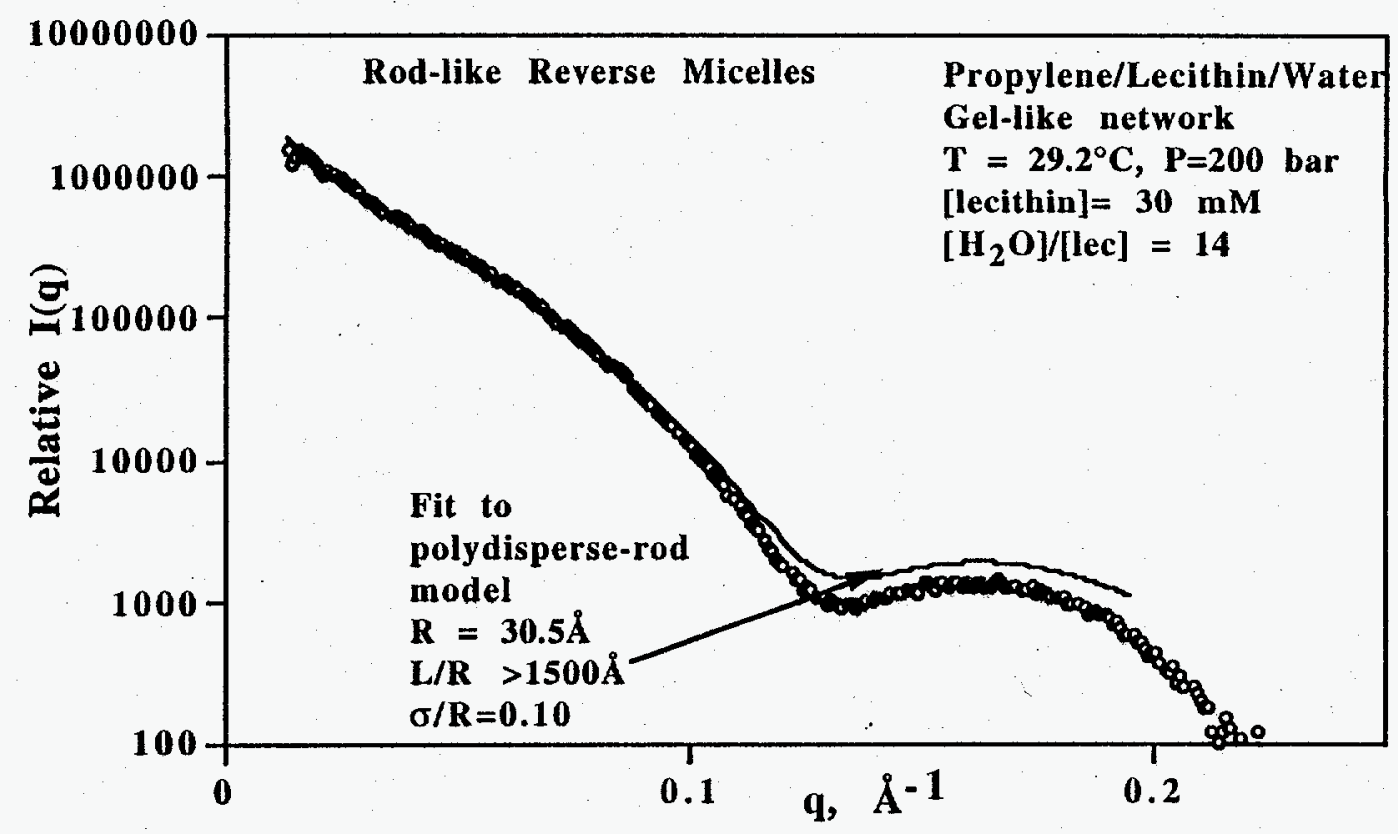

Figure 5. A rod-like surfactant aggregate in near-critical propylene.

The size and shape of surfactant aggregates are strongly dependent upon the physical properties of the surfactant molecule. The surfactant lecithin, $\mathrm{L}-\alpha$-phosphatidylcholine, forms very long, rod-like micelles in near-critical propylene under certain condition of temperature, pressure, and water concentration. Figure 5 shows SAXS data for such a system and the corresponding $P(q)$ fit to the data using a model of long rods having a small amount of polydispersity in diameter. Further supporting the proposed rod-like structure is the observation that the viscosity of this solution is several orders-of-magnitude higher than that of pure propylene at a low volume fraction of the surfactant phase, $\phi_{s}=0.06$.

\section{CONCLUSIONS}

Small-angle scattering methods provide molecular-level information about aggregation processes in supercritical fluids. Small-angle $\mathrm{x}$-ray scattering has been successfully used to study a range of different molecular aggregation processes in supercritical fluid solutions. The next generation of higher intensity synchrotron sources and neutron facilities currently under construction will offer unprecedented opportunity to probe fluid structure.

\section{Y. ACKNOWLEDGMENT.}

This research was supported by the Director, Office of Energy Research, Office of Basic Energy Sciences, Chemical Sciences Division of the U. S. Department of Energy, under contract DE-AC06-76RLO 1830.

\section{REFERENCES.}

1. Glatter, O.;Kratky, O. Small Angle X-ray Scattering, Academic Press: New York, 1982.

2. Feigin, L. A.;Svergun, D. I. Structure Analysis by Small Angle X-ray and Neutron Scattering Plenum Press: New York, 1987.

3. Kaler, E. W.; Billman, J. F.; Fulton, J. L.; Smith, R. D., J. Phys. Chem 95,1991, 458.

4. Pfund, D. M.; Zemanian, T. S.; Linehan, J. C.; Fulton, J. L.; Yonker, C. R., J. Phys Chem., 1994, submitted.

5. Londono, J. D.; Shah, V. M.; Wignall, G. D.; Cochran, H. D.; Bienkowski, P. R., J. Chem. Phys. 99,1993, 466.

6. Pfund, D. M.; Fulton, J. L.; Smith, R. D.; Carnahan, N. F.; Quintero, L.; Capel, M.; Leontaritis, K., Langmuir 9,1993, 2035. 